Operation and upgrade of diagnostic neutral beam injector RUDI at TEXTOR tokamak

A. A. Listopad, J. W. Coenen, V. I. Davydenko, P. P. Deichuli, A. A. Ivanov, V. V. Mishagin, V. Ya. Savkin, W. Schalt, B. Schweer, G. I. Shulzhenko, N. V. Stupishin, and R. Uhlemann

Citation: Review of Scientific Instruments 81, 02B104 (2010);

View online: https://doi.org/10.1063/1.3264633

View Table of Contents: http://aip.scitation.org/toc/rsi/81/2

Published by the American Institute of Physics

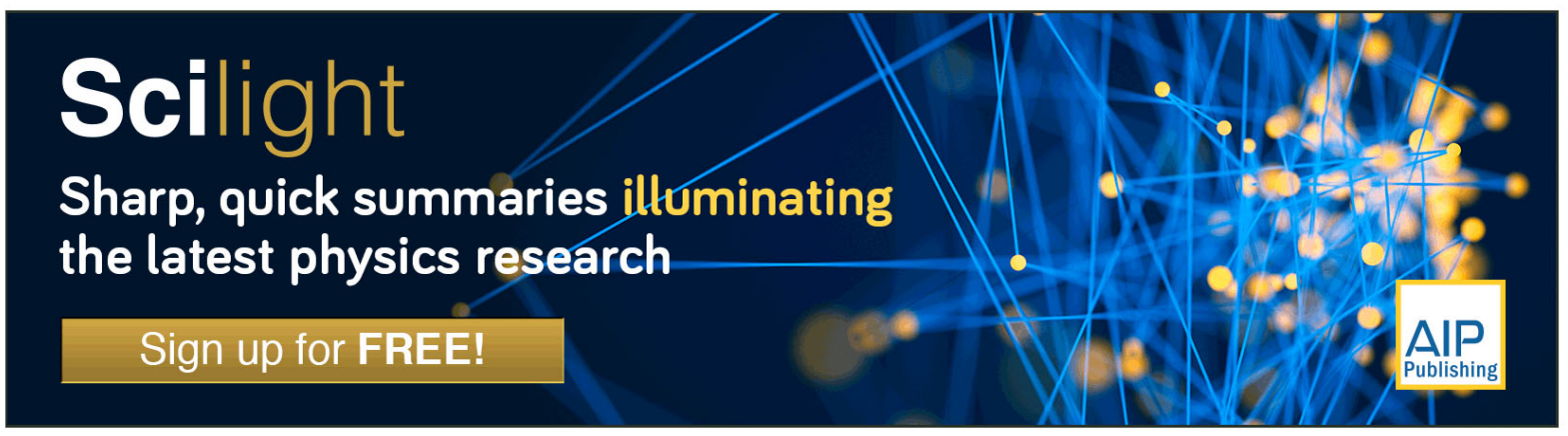




\title{
Operation and upgrade of diagnostic neutral beam injector RUDI at TEXTOR tokamak ${ }^{\text {a) }}$
}

\author{
A. A. Listopad, ${ }^{1,2, b)}$ J. W. Coenen, ${ }^{1}$ V. I. Davydenko, ${ }^{2}$ P. P. Deichuli, ${ }^{2}$ A. A. Ivanov, ${ }^{2}$ \\ V. V. Mishagin, ${ }^{2}$ V. Ya. Savkin, ${ }^{2}$ W. Schalt, ${ }^{1}$ B. Schweer, ${ }^{1}$ G. I. Shulzhenko, ${ }^{2}$ \\ N. V. Stupishin, ${ }^{2}$ and R. Uhlemann ${ }^{1}$ \\ ${ }^{1}$ Trilateral Euregio Cluster, Institut fuer Energieforschung-Plasmaphysik, Forschungszentrum Juelich GmbH, \\ Association EURATOM-FZJ, 52425 Juelich, Germany \\ ${ }^{2}$ Budker Institute of Nuclear Physics, 630090 Novosibirsk, Russian Federation
}

(Presented 22 September 2009; received 24 September 2009; accepted 5 October 2009; published online 11 February 2010)

\begin{abstract}
The status and the executing modernization of RUssian Diagnostic Injector (RUDI) are described. The ion source consists of arc plasma emitter and multiaperture four-electrode ion optical system. The present ion optical system with round beamlets is to be replaced by new slit apertures system for the reducing beam angular divergence in one direction. Due to enlarged dimensions and transparency of new ion optical system the extracted ion beam current will be by $50 \%$ increased. For the extension of beam pulse duration from $4 \mathrm{~s}$ to $8-10 \mathrm{~s}$ an optimized metal-ceramic arc-discharge channel is introduced. In the paper, the optical measurements results of beam parameters, including the profile of species distribution, scanned by custom-built multichannel spectroscope, are also presented. () 2010 American Institute of Physics. [doi:10.1063/1.3264633]
\end{abstract}

\section{INTRODUCTION}

Budker Institute of Nuclear Physics (Novosibirsk, Russia) has a serious experience in the development of neutral beam injectors for modern fusion devices. Based on hydrogen beam of RUssian Diagnostic Injector (RUDI), chargeexchange recombination spectroscopy (CXRS) diagnostic complex is used at the Tokamak EXperiment for Technology Oriented Research (TEXTOR) (Juelich, Germany) for the measurements of plasma rotation velocity and ion temperature distribution. ${ }^{1}$ The principle of charge-exchange diagnostics is based on the spectral analysis of light, emitted by plasma impurities due to interaction with the atoms of diagnostic beam. Plasma rotation velocity is evaluated from Doppler shift of light peak with respect to the unshifted reference, while ion temperature measurements are based on Doppler broadening of the spectra. Since the spatial resolution and CXRS signal level depend on diagnostic beam diameter and beam full-energy component current density, respectively, the improvements of the beam parameters mentioned are directly connected with the enhancement of diagnostic complex characteristics as a whole.

Two main steps are supposed to enhance RUDI beam parameters. To increase CXRS diagnostic signal and to improve spatial resolution a new ion-optical system with enlarged nominal beam current and reduced amount of aberrations is proposed. Since the beam duration is at the moment

\footnotetext{
a) Contributed paper, published as part of the Proceedings of the 13th International Conference on Ion Sources, Gatlinburg, Tennessee, September 2009.

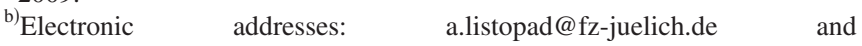

a.a.listopad@inp.nsk.su.
}

limited by $4 \mathrm{~s}$ by arc-discharge element overheating, the new metal-ceramic arc channel has been developed in order to cover $10 \mathrm{~s}$ TEXTOR discharge time.

\section{RUDI INJECTOR AT TEXTOR TOKAMAK}

To meet the requirements of the CXRS diagnostics at TEXTOR, the injector was designed to be rated at an energy of up to $50 \mathrm{keV}$ and an equivalent atomic beam current (for hydrogen) of up to $1 \mathrm{~A}$ (Fig. 1). The diagnostic beam is formed by multiaperture four-electrode ion optical system with geometrical focusing. Initially, RUDI ion source was based on rf plasma box, which consisted basically of a vacuum-tight cylindrical alumina ceramic chamber and an external rf coil.

Since the year 2006 arc-discharge plasma box with directly heated cathode is used in the ion source ${ }^{2}$ (Fig. 2). The cathode element is equipped with $\mathrm{LaB}_{6}$ stack of disks alternated by flexible washers made of thermoextended graphite. The use of $\mathrm{LaB}_{6}$ as the emitter material avoids its erosion

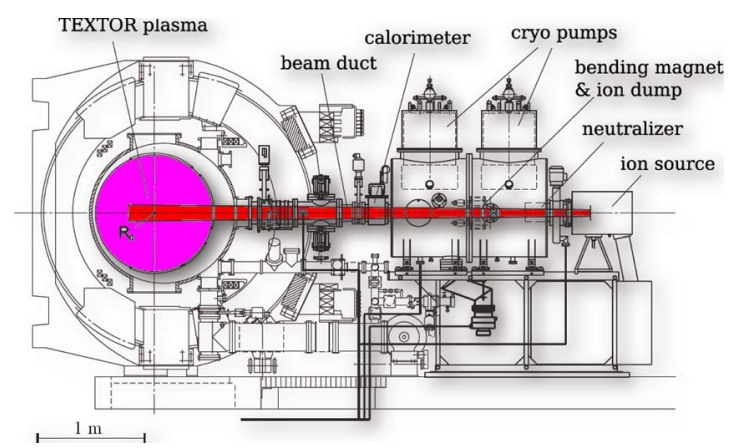

FIG. 1. (Color online) RUDI injector at TEXTOR tokamak. 


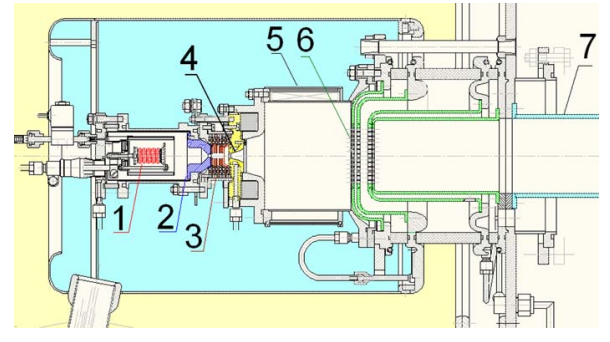

FIG. 2. (Color online) RUDI ion source overview: 1-filament heater, 2-cathode, 3-arc discharge channel, 4-anode, 5-plasma box, 6-accelerating grid system, and 7-neutralizer tube.

and provides for a long lifetime operation at working temperatures of $\sim 1650{ }^{\circ} \mathrm{C}$, with arc-discharge current of up to $300 \mathrm{~A}$.

The application of arc-discharge emitter provides high $(\sim 85 \%) \mathrm{H}^{+}$ions fraction contribution into the plasma produced. The maximal pulse duration in $5 \mathrm{~Hz} 50 \%$ : $50 \%$ modulated discharge regime is $4 \mathrm{~s}$.

\section{MULTISLIT GRID SYSTEM DESIGN}

Low angular divergency is one of the key requirements to diagnostic neutral beams. The present RUDI grid geometry with round apertures was optimized to provide the beam divergence at $0.5^{\circ}$. One of the possible approaches for further decrease in the divergence is an application of beamlets with slitlike geometry. ${ }^{3}$ In this case there are no electric field component along the slit that means low beam spread due to aberrations. The remaining divergence along the slit is essentially determined by ion temperature of plasma emitter which typically is quite low, of the order of $1 \mathrm{eV}$. Assuming the ion temperature in this range one can estimate the resulting divergence as $(\mathrm{Ti} / \mathrm{E})^{1 / 2}$, which gives $\approx 0.3^{\circ}$ for the beam protons energy of $50 \mathrm{keV}$. Among other advantages of the multislit ion-optical system geometry is its higher transparency (by $\sim 30 \%$ than that for the geometry with round beamlets) and then higher total beam current at the same grid dimensions. Some problem of multislit grids is somewhat lesser capacity of heat removal in the direction across the slits. The geometry version with round hole apertures, which is used at the moment was shown at Fig. 3, left.

In the year 2008 the decision to use a slit ion optical system in new enhanced $3 \mathrm{~A}, 8 \mathrm{~s}$ regime of RUDI operation was taken. To increase the beam current, active grid diameter was enlarged from $73 \mathrm{~mm}$ to $85 \mathrm{~mm}$. The appropriate variances of multislit ion optical system for RUDI injector were considered using an optimization by computer simulations. As a result, the version of slit grid which is shown in Fig. 3, right was chosen.
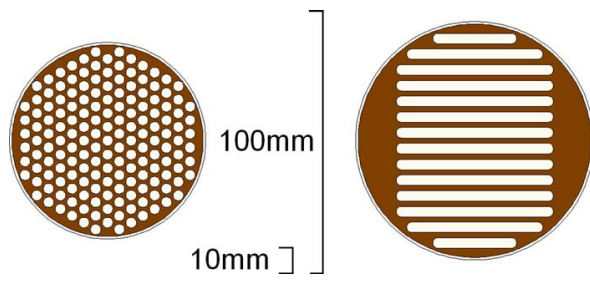

FIG. 3. (Color online) Present (left) and new (right) grid geometries.

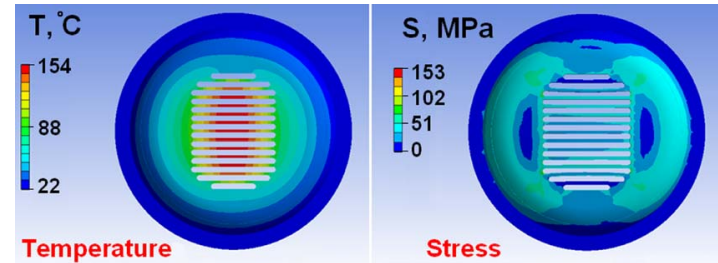

FIG. 4. (Color online) Thermal simulation of slit grid geometry.

The investigations of the grids heating influence to the ion beam formation for the geometries with $4 \mathrm{~mm}$ thickness was performed using ANSYS computer code. The power absorbed by the grid during $8 \mathrm{~s}$ beam pulse was considered 600 $\mathrm{W}$ (assuming the heating power of the order of $0.8 \%$ from total beam power ${ }^{4}$ ). The corresponding temperature rise profile and internal stress intensity distribution for the mentioned grid geometry version are presented at Fig. 4.

The obtained results of the computer simulations for the grid version presented are considered to be acceptable for accurate ion beam formation. The transparency of the geometry is $67 \%$. Further transparency increasing is not reasonable because of the reduction of the grid strength and rise of thermal deformations.

\section{MODERNIZATION OF ARC CHANNEL}

The critical question for a long pulse operation of the arc plasma source with $\mathrm{LaB}_{6}$ electron emitter is a high thermal load onto the washers in the discharge channel. At present, metal-ceramic version of the arc-discharge element with increased tolerable level of the thermal load is developed.

The view of metal-ceramic arc channel with enlarged thermal capacity is shown in Fig. 5. Thermomechanical simulations of the optimized version by ANSYS code were carried out. As a material of washers and cathode, molybdenum alloy was chosen, ceramic rings are performed of alumina oxide; the anode material is copper alloy. The conditions for simulations were the following: initial temperature of the channel elements is $22{ }^{\circ} \mathrm{C}$, power load onto each diaphragm is $300 \mathrm{~W}$ (assuming the heating power as $\sim 30 \%$ from the effective arc power which is $8 \mathrm{~kW}$ ), convective cooling rate of the diaphragms by water flowing at periphery is $16000 \mathrm{~W} / \mathrm{m}^{2} \mathrm{~K}$, pulse duration is $8 \mathrm{~s}$.

It was found that the temperature global maximum of the channel elements is achieved at inner surface of the fifth diaphragm. The dependence of global maximum of tempera-

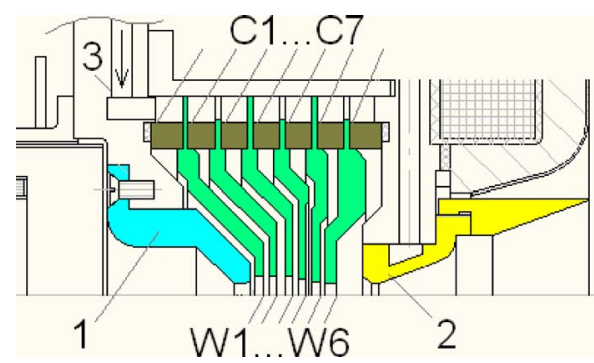

FIG. 5. (Color online) New metal-ceramic discharge element: 1-cathode, 2-anode, 3-water channel, C1C.C7-ceramic insulators, and

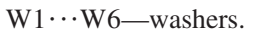




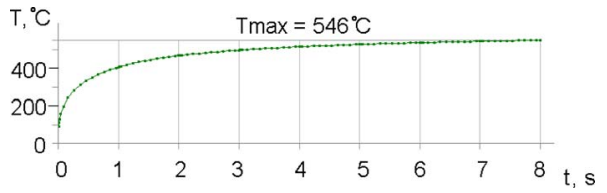

FIG. 6. (Color online) Global maximum temperature dynamics.

ture on time during the discharge pulse is presented in Fig. 6 . The results of the simulations demonstrate that in $8 \mathrm{~s}$ discharge pulse steady-state temperature regime of the discharge channel elements is practically achieved. The temperature of diaphragms near the water channel is lower than $50{ }^{\circ} \mathrm{C}$, near seals-lower than $70{ }^{\circ} \mathrm{C}$, temperature of ceramic rings-lower than $60^{\circ} \mathrm{C}$ after $8 \mathrm{~s}$ shot.

Maximal stress is obtained on the fifth diaphragm (860 MPa). Maximal stresses on ceramics are achieved on the rings 6 and 7 (120 and $100 \mathrm{MPa}$, accordingly).

The obtained values of elements temperature, deformations, and stresses are well appropriate for reliable operation.

\section{OPTICAL DIAGNOSTIC SETUP}

In the frames of RUDI modernization the new beam diagnostic setup has designed (Fig. 7). The complex includes multichannel spectroscope for beam spectra profile investigation. The device was developed for the monitoring of beam profiles of each fraction (full-, half-, and third-energy components, and water contribution as well). It also gets one the knowledge about species composition along the beam profile. The principle of the diagnostics is based on $\mathrm{H}_{\alpha}$ Doppler shift mechanism, ${ }^{5}$ due to that the spectrum of the beam researched represents a set of shifted $\mathrm{H}_{\alpha}$ bright lines (typically, it consists of four peaks according to the beam species amount), and also unshifted $\mathrm{H}_{\alpha}$ line, that corresponds to the background gas.

The device is mounted inside the injector vacuum vessel at $\sim 2 \mathrm{~m}$ from the grid system. It has seven channels to scan the beam profile with the step of $1.5 \mathrm{~cm}$. The angle of observation is $45^{\circ}$ to the beam axis.

Due to the special requirements, the optical scheme of the endoscope with close first lens position to the beam axis was designed in order to get the maximum of the beam light intensity. The light collected is then transported out of the vacuum vessel. The beam optical image is built with magnification $\sim 10$ at the fiber matrix plane. The example of beam spectra measurements is presented in Fig. 8.

For high-resolution beam profile scanning, chargecoupled device $(\mathrm{CCD})$ camera is planned. Equipped with

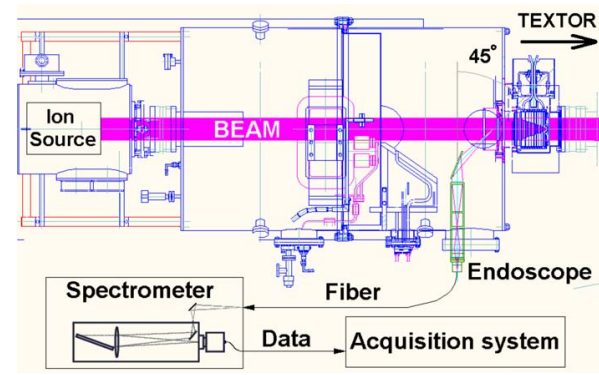

FIG. 7. (Color online) RUDI optical diagnostic setup.

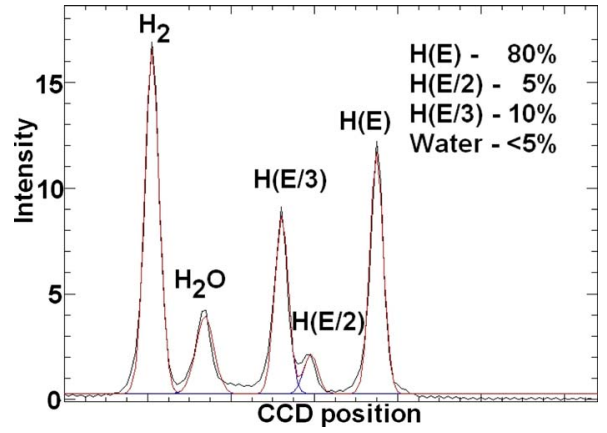

FIG. 8. (Color online) RUDI beam spectra image.

high-aperture optics the device will allow one to achieve the beam images in the visible range of the spectra. Using the filters of respective bright lines one can monitor the beam impurities such as carbon, copper, molybdenum, and oxygen as well.

For the beam divergence measurements via $\mathrm{H}_{\alpha}$ light peaks broadening the spectrometer with high spatial resolution will be used. Observing the beam in perpendicular direction, the peak of $\mathrm{H}_{\alpha}$ line has the spread due to transverse velocities. Assuming the beam divergence in the range of $0.5^{\circ}$ at $50 \mathrm{keV}$ Hydrogen atoms energy the resulting broadening $(\Delta \lambda / \lambda)$ is $\sim 10^{-4}$. That means for the measurements with beam divergence resolution $\sim 0,1^{\circ}$ the setup with resolution $\sim 0,1 \AA$ /pixel is required. Having the observation angle deviation about $5^{\circ}$ from perpendicular direction one can resolve the peaks broadening separately for each beam fraction. Further deviation of angle is not reasonable because of the effect of longitudinal broadening due to particles energy dispersion.

\section{SUMMARY}

The RUDI neutral beam injector provides the steady measurements of TEXTOR plasma parameters. The measures to increase the beam parameters have been taken and new optical diagnostic setup has been developed. At present, new slit ion optical system and plasma emitter with increased diameter are being fabricated, the first version of metalceramic arc-discharge channel is designed and experimental investigations at the test bed of Budker Institute are been prepared.

Multichannel spectroscope is fabricated, adapted to the spectrometer installed and present TEXTOR data acquisition system. In the future, the high-resolution spectrometer is planned for beam transverse velocities monitoring by $\mathrm{H}_{\alpha}$ peaks broadening.

${ }^{1}$ J. W. Coenen, M. Clever, U. Samm, O. Schmitz, B. Schweer, and B. Unterberg, "Charge exchange spectroscopy on a hydrogen diagnostic beam for measurements of impurity rotation and the radial electric field at the Tokamak TEXTOR,” Rev. Sci. Instrum. (to be published).

${ }^{2}$ A. A. Ivanov, V. I. Davydenko, P. P. Deichuli, G. I. Shulzhenko, and N. V. Stupishin, Rev. Sci. Instrum. 79, 02 C103 (2008).

${ }^{3}$ V. I. Davydenko, A. A. Ivanov, I. V. Shikhovtsev, and A. V. Sorokin, Rev. Sci. Instrum. 79, 02B720 (2008).

${ }^{4}$ A. D. Beklemishev, V. I. Davydenko, A. A. Ivanov, and A. A. Podyminogin, Rev. Sci. Instrum. 69, 2007 (1998).

${ }^{5}$ S. J. Yoo, H. L. Yang, and S. M. Hwang, J. Korean Phys. Soc. 35, 315 (1999). 